\title{
Turning a conventional camera into a 3D camera with an add-on
}

\author{
P.Trouvé-Peloux ${ }^{1}{ }^{*}$, J. Sabater ${ }^{2}$, A. Bernard-Brunel ${ }^{1}$, F. Champagnat ${ }^{1}$, G. Le Besnerais ${ }^{1}$, \\ AND T. AVIGNON ${ }^{2}$ \\ ${ }^{1}$ ONERA-The French Aerospace Lab, FR-91761, Palaiseau, France. \\ ${ }^{2}$ Institut d'Optique Graduate School, 2 Avenue Augustin Fresnel, RD128 91127n Palaiseau, France. \\ ${ }^{*}$ Corresponding author: pauline.trouve@onera.fr
}

Compiled May 18, 2018

\begin{abstract}
We propose to add an optical component in front of a conventional camera to improve depth estimation performance of Depth from Defocus (DFD), an approach based on the relation between defocus blur and depth. The add-on overcomes ambiguity and dead zone, which are the fundamental limitations of DFD with a conventional camera, by adding an optical aberration to the whole system which makes the blur unambiguous and measurable for each depth. We look into two optical components: the first one adds astigmatism and the other one chromatic aberration. In both cases we present the principle of the add-on and experimental validations on real prototypes. (c) 2018 Optical Society of America
\end{abstract}

OCIS codes: (110.6880) Three-dimensional acquisition; (110.1758) Computational imaging; (080.1005) Aberration expansions.

http://dx.doi.org/10.1364/ao.XX.XXXXXX

\section{INTRODUCTION}

The development of 3D applications in domotic, robotic or augmented reality, leads to a growing interest for compact 3D cameras. Most 3D devices are based on stereoscopy, a well know approach for depth estimation based on the measure of disparity between images from different cameras [1,2]. Recent systems also project an infrared pattern on the scene to counteract the potential lack of texture [3]. However, stereoscopy requires two cameras, increasing the size of the system and leading to calibration issues. An interesting alternate option is Depth from Defocus (DFD) [4]. DFD exploits the relation between depth and defocus blur, and requires a single lens only, thus opening the way to very compact 3D cameras. While some DFD approaches require multiple images with various optical parameters $[5,6]$, we focus here on Single Image DFD (SIDFD) which is more suited to dynamic scenes capture. However, as pointed out in [8], SIDFD with a conventional lens suffers from depth ambiguity (a similar blur amount can be measured in front or behind the camera in-focus plane), and from a "dead zone" within the Depth of Field (DoF) of the camera where no blur variation can be measured. Specific camera settings allow to mitigate these limitations, e.g. focusing at infinity to avoid blur ambiguity and choosing a large camera aperture do reduce the DoF. However these settings induce a large defocus blur, leading to a significant loss of depth estimation accuracy, as demonstrated in [8].

In this paper, we propose a cheap and user friendly approach to give a $3 \mathrm{D}$ ability to a conventional camera. It consists in mounting an optical component, which we call an "add-on", in front of the lens. This component adds an aberration which reinforces the variability of the defocus blur with respect to depth, thus improving DFD performance. We describe two different components, based on two different optical aberrations: astigmatism and chromatic aberration. We show that both type of components provide a solution to avoid the dead zone and depth ambiguity of SIDFD. Moreover, they imply only a small additional cost and require the tuning of a limited number of parameters. For both approaches, we present a theoretical analysis based on a simple model of the combination of a conventional lens with the add-on. We show experimental results on several types of cameras: an industrial camera, a reflex camera and a smartphone. We evaluate the DFD accuracy with and without the add-on and discuss the potential field of application of the proposed technique. We conclude that both systems improve the DFD estimation process with respect to a conventional camera and that they have different domains of application

\section{A. State of the art}

Adding optical components to a conventional camera to improve the performance of the overall system is a widespread approach in computational imaging. In the context of 3D imaging, amplitude and phase masks have been proposed to increase the depth dependence of the PSF [9-11] and [12, 13]. For optimal performance, phase masks have to be put at the principal plane or the Fourier plane of the lens. However, these planes are not precisely known nor easy to access for a commercial lens, lead- 
ing to non negligible manufacturing costs. Amplitude masks are placed at the camera aperture which is also not always easy to access. Besides, amplitude coded apertures generally do not provide a solution to the depth ambiguity and dead zone of DFD - except for asymmetric apertures [11] which avoid ambiguity.

In previous works $[7,23]$ we have demonstrated the interest of using a lens with a chromatic aberration designed so that three blurs associated to the RGB channels can be measured at each depth. This approach resolves the aforementioned DFD limitations, and allows to limit the amount of defocus blur (at least in one of the three channels) over a wide range of depths. As a result, the accuracy of the DFD was significantly improved with respect to a camera with a conventional lens. However, this approach relies on a specific chromatic lens and thus implies a complex lens design optimization and costs associated to the realisation of tailored optical elements. Besides, this approach can be use only when changing the camera lens is possible.

In the field of particle 3D localisation in microscopy, some authors have proposed to add a cylinder lens in front of the CCD sensor of an inverted microscope[14]. The cylinder lens acts as a plane-parallel plate in one plane and changes the focal length of the optical system in the perpendicular plane. In doing so, some astigmatism is added to the system and the PSF becomes elliptical, with major axes having a length variable with the depth. By looking at the shape of the elliptical PSF produced on the image plane by a particle one can deduce its 3D position. To the best of our knowledge this approach has never been used for DFD application on natural scenes.

In this paper we propose to add an optical component in front of a conventional camera to improve its 3D measurement ability. The proposed add-ons are simple to realise and can easily be removed from the conventional camera if the user does not need a 3D acquisition. We have looked into two types of add-on: one adds astigmatism thanks to cylindrical lens and the other one adds chromatic aberration with an afocal doublet. Prototypes of the two add-ons have been realized and evaluated through depth estimation experiments using either an industrial camera, a reflex camera or a smartphone. We provide experimental comparison of DFD performance of the system with and without the add-on and discuss the field of applications of each approach.

\section{B. Paper organization}

The following sections are dedicated to the cylinder (Section 2) and chromatic (Section 3) components. For each add-on we present its principle, a geometric model that emphasizes the parameter governing the defocus blur and an experimental performance evaluation. Finally, we discuss their respective field of applications. We conclude in Section 4.

\section{CYLINDER ADD-ON}

\section{A. Principle}

Figure 1(a) illustrates the shape of a cylinder lens. In one plane, referred to as the $O x z$ plane, this lens acts as a simple planeparallel plate while in the perpendicular plane, referred to as the Oyz plane, it acts as a conventional spherical lens of radius of curvature $R$. Hence this lens introduces a different position of the focus plane depending on the ray direction, which causes astigmatism. Adding a cylinder lens in front of a conventional camera then leads to an elliptical PSF whose lengths of the main axes depend on depth. Cylinder lenses have been proposed in the literature for particle 3D velocimetry [14], we study here their use for SIDFD on natural scenes.

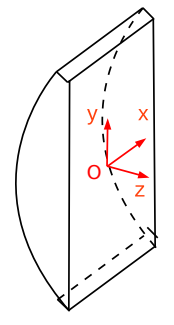

(a)

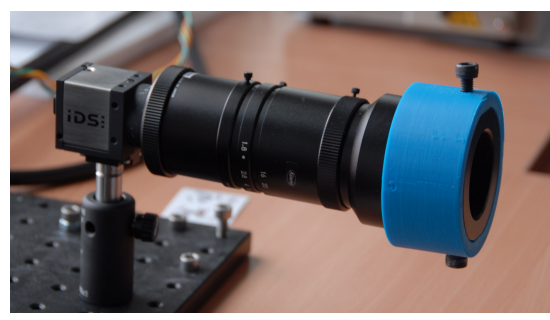

(b)
Fig. 1. (a) Cylinder lens. (b) Industrial camera with the cylinder add-on.

\section{B. PSF model}

Appendix A presents the model of a lens combining a conventional optical system with an add-on, assuming a Gaussian PSF. In this section, we use this model to simulate the PSF of an optical system made of a cylinder lens placed in front of a conventional camera.

The cylinder add-on is assumed to be a perfect thin lens with a focal length $f_{a}^{\prime}$ in the $O y z$ plane and an infinite focal length in the $O x z$ plane. The value of $f_{a}^{\prime}$ is then:

$$
f_{a}^{\prime}=\frac{R}{n-1}
$$

where $n$ is the refractive index of the material of the cylinder lens. Here we neglect both the add-on width and the variation of $n$ with respect to wavelength. As the focal length varies in the $O x z$ and $O y z$ planes, we propose to model the PSF of the cylinder add-on as an anisotropic Gaussian PSF function :

$$
h(x, y, z)=\frac{1}{2 \pi \sigma_{x} \sigma_{y}} \exp \left(-\frac{x^{2}}{2 \sigma_{x}^{2}}-\frac{y^{2}}{2 \sigma_{y}^{2}}\right),
$$

where $\sigma_{x}$ and $\sigma_{y}$ are defined by equation (7) in Appendix A with $f_{a}^{\prime}=\infty$ in the $O x z$ plane and $f_{a}^{\prime}$ given by equation (1) in the Oyz plane. Figure 2 shows an example of the variation of $\sigma_{x}$ and $\sigma_{y}$ with respect to depth for a simulated optical system with $f^{\prime}=35 \mathrm{~mm}, f_{\#}=4, t_{p x}=9 \mu \mathrm{m}$ and the in-focus plane in the undisturbed direction placed at $1.5 \mathrm{~m}$. The working wavelength is fixed at $530 \mathrm{~nm}$ corresponding to the central wavelength of the green channel spectral response of a commercial sensor[18]. The cylinder add-on has an index of $n=1.5$ and is fixed at a distance $|e|$ from the conventional lens arbitrarily put at $15 \mathrm{~mm}$ (see Figure 17). Figure 2 shows that the cylinder add-on clearly creates different in-focus plane positions in the $O x z$ and $O y z$ planes. The PSF parameters have been traced for three values of the radius or curvature $R=1.5,2$ and $4 \mathrm{~m}$ : a decrease of $R$ leads to an increase of the distance between the $O x z$ and $O y z$ in-focus planes. Hence $R$ appears as the main parameter for tuning the amount of astigmatism of the system.

\section{Benefits and limitations}

Figure 2 shows that, for a given value of $R$, there is a single doublet of $\left\{\sigma_{x}, \sigma_{y}\right\}$ per depth, thus the ambiguity in depth estimation is resolved. Besides, if no blur variation can be observed in one axis, due to the DoF in this direction, there is always a blur variation that can be observed in the other direction. Hence, the cylinder add-on seems to overcome the main limitations of conventional lens SIDFD. However it is implicitly assumed that both $\sigma_{x}$ and $\sigma_{y}$ are observable on the scene. This is not true for 


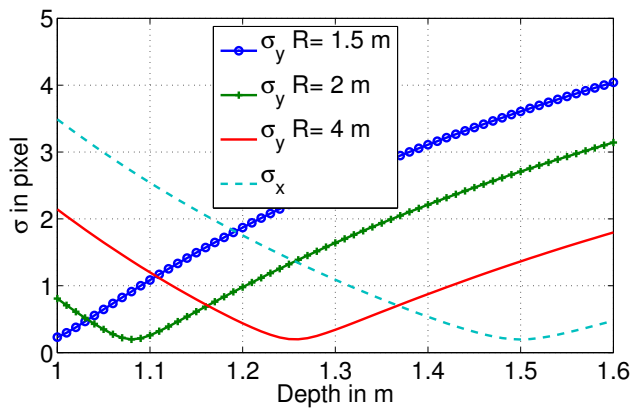

Fig. 2. Variation of PSF standard deviation in the $O x z$ and $O y z$ planes for different values of the radius of curvature of the cylinder lens. The main parameters of the conventional lens are $f^{\prime}=35 \mathrm{~mm}, f_{\#}=4$ and $n=1.5 @ 530 \mathrm{~nm}$ and the in-focus plane in the $O x z$ plane is fixed at $1.5 \mathrm{~m}$.

mono-directional scenes, ie. edge regions where the intensity is invariant along one direction and follows a $1 \mathrm{D}$ profile on the orthogonal direction parametrized by the angle $\theta$ in Figure 3 (a). In this case, the observed blur for mono-directional scenes corresponds to the projection of the PSF on the axis parallel to direction $\theta$ of the 1D profile. The projected blur size $\sigma_{\text {proj }}$ is then defined as :

$$
\sigma_{\text {proj }}=\sqrt{\left(\sigma_{x} \cos \theta\right)^{2}+\left(\sigma_{y} \sin \theta\right)^{2}} .
$$

Figure 3(b) shows the variation of $\sigma_{\text {proj }}$ with respect to depth for various values of $\theta$ and for the system simulated in Figure 2 with $\mathrm{R}=1 \mathrm{~m}$. It shows that measuring the projected blur size does not resolves ambiguous depth estimations anymore.

(a)
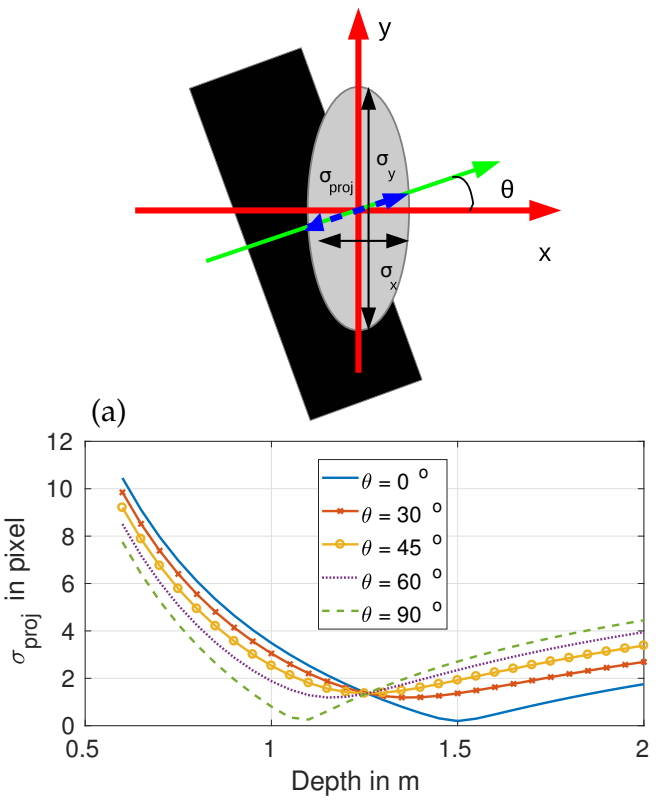

(b)

Fig. 3. (a) Case of a mono-directional scene. The gray ellipse pictures the defocus blur. (b) Variation of $\sigma_{p r o j}$ with respect to depth, for various orientations of the scene $\theta$. The optical system is the same as the one in Figure 2, with $R=1 \mathrm{~m}$.

Note that the proposed add-on usually brings other aberrations in addition to astigmatism. Simulations on an optical design software (assuming a perfect lens for the conventional camera) show that the main unwanted aberration is distortion. This aberration had no noticeable effect in our experiments, but if it had been the case it could have been corrected by distortion calibration and dedicated processing. Other unwanted aberrations can be avoided by a proper optimization of the flat surfaces of the add-on.

In summary, the add-on cylinder offers a way to avoid the classic limitations of SIDFD for scenes with well-distributed gradient orientations. However, it does not bring any gain on one-directional patterns, which appear frequently in man-made environment and particularly in indoor scenes. In such situations, practical use of the add-on cylinder could require additional texture, for instance by using speckle-like structured illumination. Moreover, it should be noted that astigmatism reduces the quality of the image: a deconvolution step can restore it partially, depending on the noise level, at the cost of a significant calculation load however.

\section{Experimental validation}

\section{D.1. Settings}

In the following we experiment the cylinder add-on mounted on an industrial camera, on scenes which are assumed to be sufficiently isotropic to conduct depth estimation. We are interested in depth estimation in the range between $1 \mathrm{~m}$ and $1.6 \mathrm{~m}$. We use a conventional zoom lens, with continuous variation of the f-number and the focal length. The sensor size is of $1 / 1.8$ " and the pixel size $t_{p x}$ is of $4.5 \mu \mathrm{m}$. The add-on material is in N-BK7 whose refractive index is $n=1.52$ at $530 \mathrm{~nm}$. Depth is estimated with and without the add-on, on the green channel extracted from the raw image without interpolation (as a result, the resolution is divided by two and the equivalent pixel size is $9 \mu \mathrm{m}$ ). The focal length and the f-number are approximatively placed at $35 \mathrm{~mm}$ and 4 in order to get a defocused PSF of width $\sigma_{\text {def }}$ between 0 to 3 pixels within the depth range. The focus plane of the conventional lens is fixed at the largest distance of the depth range, ie $1.6 \mathrm{~m}$, to avoid ambiguity when depth estimation is conducted without the add-on. We choose a cylinder lens of radius $2 \mathrm{~m}$, so that both in-focus planes in the $O x z$ and $O y z$ planes are contained in the chosen depth range (between $1 \mathrm{~m}$ and $1.6 \mathrm{~m}$ ). Figure 1(b) shows a picture of the resulting optical system.

\section{D.2. Depth estimation algorithm}

We estimate the depth using the method described in [15] (see Appendix B.A), applied on the green channel extracted from the raw data. For each image patch, a PSF is selected by maximisation of a generalized likelihood among a set of potential PSFs. In practice, this amounts to the optimization of a local cost function over two parameters, the signal to noise ratio (SNR) and the PSF index. Each PSF is related to a depth through a calibration procedure described in the next section.

\section{D.3. PSF calibration}

To get a set of potential PSFs as close as possible to the true ones, we calibrate the PSFs from 1 to $1.6 \mathrm{~m}$ with a step of $1 \mathrm{~cm}$. Calibration is conducted with and without the add-on using acquisitions of a textured pattern, following the method of [16]. Figure 4 shows examples of calibrated PSFs at different depths with the add-on. As expected, PSFs have an elliptical shape. Note that they are tilted by an angle approximately equal to $45^{\circ}$, revealing that the major axes of the cylinder lens are tilted with respect to the sensor axes. 


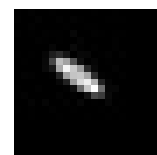

(a)

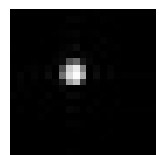

(b)

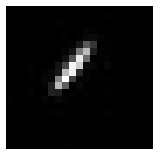

(c)
Fig. 4. PSFs at 3 depths: (a) $1 \mathrm{~m}$ (b) $1.2 \mathrm{~m}$ (c) $1.6 \mathrm{~m}$ with the cylinder add-on placed in front of a conventional camera.

We experimentally measure the standard deviation $\sigma_{x, \exp }$ and $\sigma_{y, \exp }$ of the blur in both major directions of the cylinder lens. To do so we use a simple knife-edge blur estimation, proposed in [17], on black and white targets positioned at different distances from the camera, having the same orientations as the cylinder major axes. Direct comparison of these experimental values with theoretical $\sigma_{x}$ and $\sigma_{y}$ is not straightforward. Indeed we use a zoom lens with a focal length and an aperture that can vary continuously, so the values of $f^{\prime}$ and $f_{\#}$ which have been used are only approximation of the true values. Besides, we model the add-on and the conventional lens as perfect thin lenses while in practice they have a physical width. However we show in Figure 5 that one can empirically find a set of parameters $f_{\#}, e$ and $f^{\prime}$, close to the expected values, that gives a good match between theoretical standard deviation and experimental measures of the blur. For this example, those parameters are $f^{\prime}=34.5 \mathrm{~mm}$, $f_{\#}=4.5$ and $|e|=13 \mathrm{~cm}$. We can conclude that the simple anisotropic Gaussian PSF model deduced from Appendix A and Equation (2) provides an efficient model of the hybrid optical system combining a cylinder add-on and a conventional system.

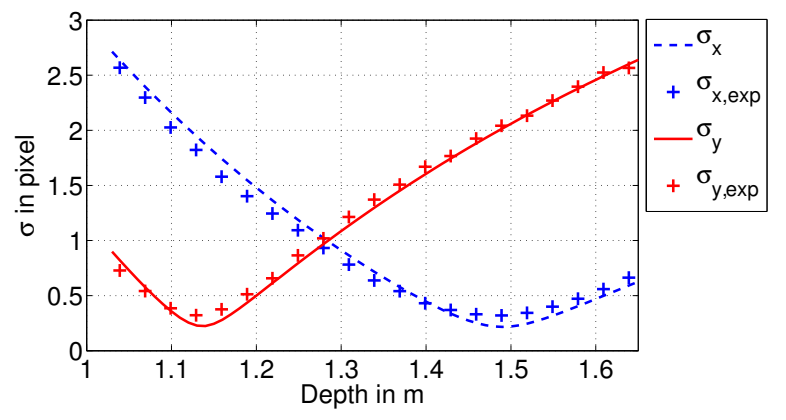

Fig. 5. Comparison of theoretical and experimental values of $\sigma_{x}$ and $\sigma_{y}$. Theoretical parameters are $f^{\prime}=34.5 \mathrm{~mm}, f_{\#}=4.5$, $|e|=13 \mathrm{~cm}$ and $R=2 \mathrm{~m}$ and in-focus plane at $1.5 \mathrm{~m}$.

\section{D.4. Experimental depth estimation}

Different textured scenes are put at a known distance from the camera. At each depth, a depth map is estimated within a patch of $25 \times 25$ pixels using the algorithm described in Appendix B. Figure 6 shows the mean depth estimation and standard deviation with respect to the ground truth, inside a centred region of the depth map corresponding to a square of $15 \times 15$ patches. This experiment is conducted both with and without the cylinder add-on. For both sets of measured values, we have removed $5 \%$ of the maximal and minimal values in order to remove outliers in the statistics. Figure 6 shows that in the range $1 \mathrm{~m}$ to $1.3 \mathrm{~m}$ both configurations (with and without the addon) show unbiased results with a limited standard deviation.
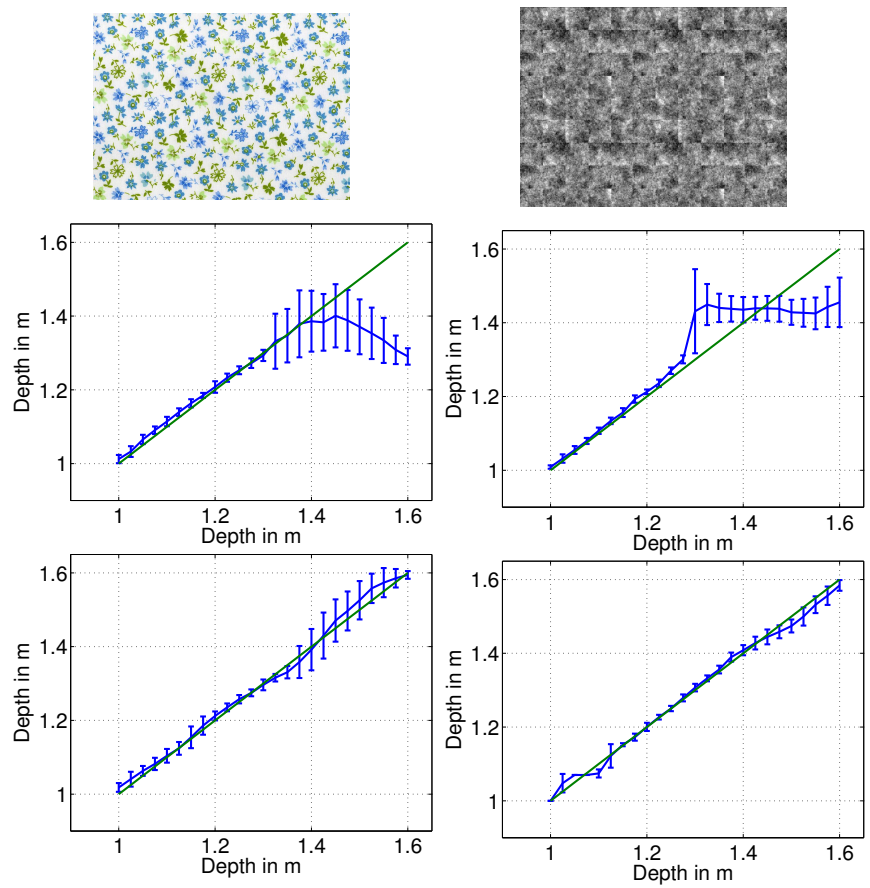

Fig. 6. Depth estimation statistical result. First row: pattern used to estimate depth. Second row: without the cylinder addon. Third row: with the cylinder add-on.

More precisely, the mean square error (MSE) is about $2 \mathrm{~cm}$ in both cases. After this depth, depth estimation without the addon (middle row of Figure 6) shows large errors with a MSE varying from $8 \mathrm{~cm}$ to $15 \mathrm{~cm}$. Indeed, objects after $1.3 \mathrm{~m}$ are sharp because of the camera depth of field, so no blur variation can be measured. In contrast, with the cylinder add-on, the MSE stays below $6 \mathrm{~cm}$ in the range $1.4 \mathrm{~m}$ to $1.6 \mathrm{~m}$ (last row of Figure 6). We conclude that on such textures patterns, the cylinder add-on brings a significant gain in accuracy of the depth estimation.

\section{D.5. Depth maps}

Figure 7 shows two depth maps obtained with natural scenes in the range 1 to $1.6 \mathrm{~m}$. Depth estimation is conducted on patches scanning the whole image with a shift of one pixel, thus with maximal overlapping between neighbouring patches. The depth of each object of the scene is correctly estimated if there is a sufficiently contrasted texture. Note however that within regions with mono-directional gradients, such as the region on the top of the cup in (a) there are aberrated results.

\section{CHROMATIC ADD-ON}

\section{A. Principle}

In this Section, we consider another add-on made of a chromatic doublet illustrated in Figure 8. The glasses of each lens are chosen to have an identical refractive index at a nominal wavelength in the visible, but various refractive index at other wavelengths. Thus the add-on acts as a plane-parallel plate for the nominal wavelength, and has a non zero power for the other wavelengths in the visible spectrum, which causes the chromatic aberration. The parameters of the add-on are the two glasses and the radius of curvature $R$ of their common surface. Let $n_{1}(\lambda)$ (resp. $n_{2}(\lambda)$ ) be the refractive index of the first (resp. second) glass at the wavelength $\lambda$. Using a thin lens approximation, the focal length 


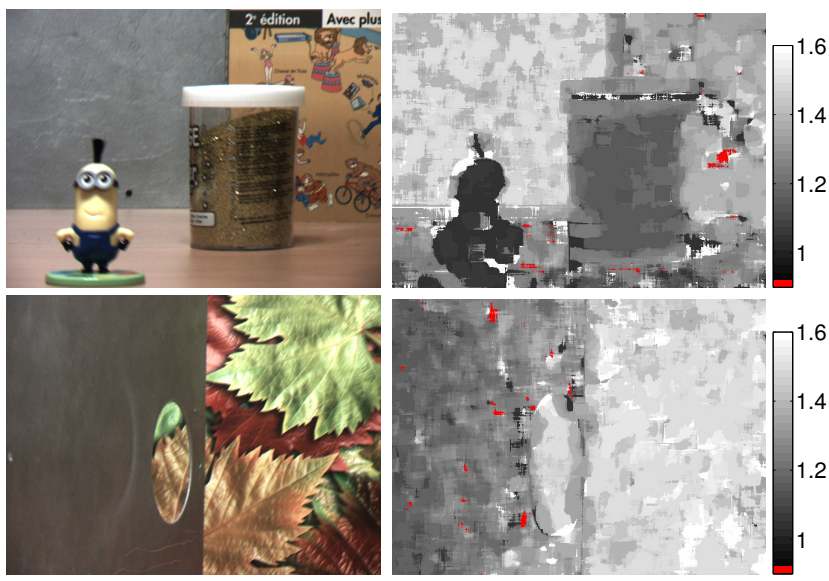

Fig. 7. Experimental scenes and corresponding depth maps using the cylinder add-on. First scene (Top) is made of four objects located at $1.6 \mathrm{~m}$ (wall), $1.38 \mathrm{~m}$ (book), $1.2 \mathrm{~m}$ (pot), $1.03 \mathrm{~m}$ (doll). Second scene (Bottom) is made from a tilted metallic plate with depth ranging from $1.1 \mathrm{~m}$ to $1.4 \mathrm{~m}$ (the hole is at $1.4 \mathrm{~m}$ ) and a picture of leaves on the wall behind at $1.5 \mathrm{~m}$. Red is for textureless regions corresponding to $\widehat{\alpha}>0.1$ (see Appendix B).

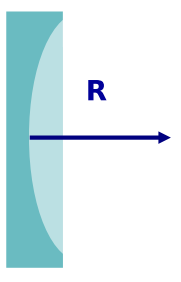

(a)

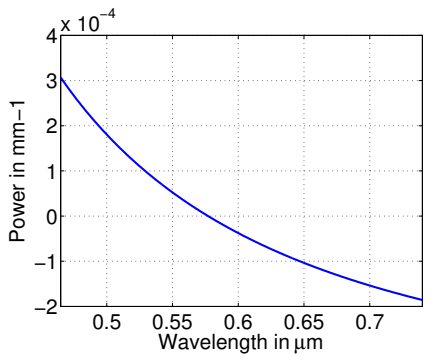

(b)
Fig. 8. (a) Illustration of the chromatic add-on. (b) Variation of the add-on power $P_{a}$ with respect to the wavelength with $\mathrm{N}-\mathrm{F} 2, \mathrm{~N}-\mathrm{SK} 16$ glasses and $\mathrm{R}=20 \mathrm{~mm}$.

of the add-on writes:

$$
\frac{1}{f_{a}^{\prime}(\lambda)}=P_{a}(\lambda)=\frac{n_{2}(\lambda)-n_{1}(\lambda)}{R} .
$$

Figure 8(b) shows an example of power variation of the doublet with respect to the wavelength. The doublet is made of the glasses N-SK16 and N-F2 which have the same refractive index at $577 \mathrm{~nm}$, with $R=20 \mathrm{~mm}$. As expected, the power of the doublet is null at the nominal wavelength while it is positive or negative at other wavelengths, which creates chromatic aberration. This figure shows that the optical component acts as a plane-parallel plate for the green channel of the color camera[18]. Hence the component does not change the main camera parameters for this channel. Note that variation of the refractive index with respect to the wavelength is obtained using Sellmeier equation with parameter from the SHOTT glass catalog.

\section{B. PSF model}

The proposed PSFs model of an hybrid optical system made of a chromatic add-on and a conventional lens, is directly based on the model described in Appendix A. Each color channel has isotropic Gaussian PSF parametrized by a standard deviation $\sigma_{c}$ defined by Equation (7) with $f_{a}^{\prime}$ defined for each color channel using Equation (4). Note that in order to take into account the intra-channel chromatic aberration, a weighted average of $\sigma$ is calculated with weights corresponding to the spectrum of each color channel. In the remaining of the paper, the R,G and $B$ spectra are simply modeled using Gaussian functions, with central wavelength and standard deviation respectively equal to $(610 \mathrm{~nm}, 55 \mathrm{~nm}),(530 \mathrm{~nm}, 35 \mathrm{~nm})$ and $(470 \mathrm{~nm}, 30 \mathrm{~nm})$ to be globally consistent with the spectra given in [18]. Figure 9 shows a model of the variation of the PSF standard deviation with respect to depth for the RGB channels and three values of $R: 3$, 10 and $300 \mathrm{~m}$. The conventional lens main parameters are an aperture of $35 \mathrm{~mm}$ with $f_{\#}=4$ and a pixel size of $12 \mu \mathrm{m}$. The in-focus plane is at $3.6 \mathrm{~m}$ for the wavelength $532 \mathrm{~nm}$. Figure 9 illustrates that the radius $R$ is the main parameter from which the amount of chromatic aberration can be set. A decrease of $R$ leads to an increase of chromatic aberration. Our aim here is tune the chromatic aberration to have maximally separated color channel DoFs in the range 1 to $5 \mathrm{~m}$. On the other hand, as shown in Figure 9 for $R=3 \mathrm{~mm}$, too much chromatic aberration leads to higher PSF standard deviations and thus to a decrease of image quality. Given these considerations, for this example, the value $R=10 \mathrm{~mm}$ appears as a reasonable choice.

\section{Benefits and limitations}

As discussed in [7] the main advantage of chromatic aberration is that both depth ambiguity and dead zone are avoided, thanks to the separation of the R,G and B in-focus planes. This approach assumes that the scene and illumination spectra are spread enough to have a sufficient SNR in the three R,G,B channels. However this assumption is usually fulfilled for natural scenes and illumination.

Now consider the problem of maintaining a good image quality with a chromatic add-on such as the one proposed in this article. First, it yields an increase of both lateral and longitudinal chromatic aberrations. While the longitudinal chromatic aberration can be usefull to estimate depth, the lateral ones induces a scale factor between the R,G and B color channels. Such an effect can be numerically corrected by warping two channels onto the third thanks to a planar homography model. Several efficient techniques are known for such operation. Second, the longitudinal chromatic aberration induces spectrally varying defocus blur. This can be partially corrected using high frequency transfer as proposed in [7], a technique which is computationally very efficient. Intra-channel chromatic aberration can also occur and has to be corrected by deconvolution, using the calibrated in-focus PSF of each channel.

Finally, as for the cylinder lens, the add-on can bring aberrations other than chromatic. Simulations conducted on an optical design software (assuming a perfect lens for the conventional camera) shows that the main unwanted aberration is spherical aberration. This aberration had no noticeable effects in our experiments, however it could be reduced if necessary by optimization of the flat surfaces of the add-on.

\section{Experimental validations}

\section{D.1. Settings}

We conduct depth estimation in the range 1-5 $\mathrm{m}$ with a camera Nikon D200 having a lens with fixed focal length of $35 \mathrm{~mm}$ and a pixel size of $6 \mu \mathrm{m}$ and $f_{\#}=4$. Depth is estimated on the color channels extracted from the raw data without interpolation (ie 
the color channel resolution is divided by two and the pixel size is assumed to be $12 \mu \mathrm{m}$ ). For experiments conducted without the add-on the in-focus plane is put at infinity in order to avoid ambiguity in depth estimation with the conventional lens only. With the add-on the in-focus plane is fixed on the green channel at $3.6 \mathrm{~m}$. The add-on is made of N-SK16 and N-F2 lenses. We built an add-on with $R=10 \mathrm{~mm}$ and fixed it in front of a D200 Nikon camera, with a lens of $35 \mathrm{~mm}$, as shown in Figure 10.
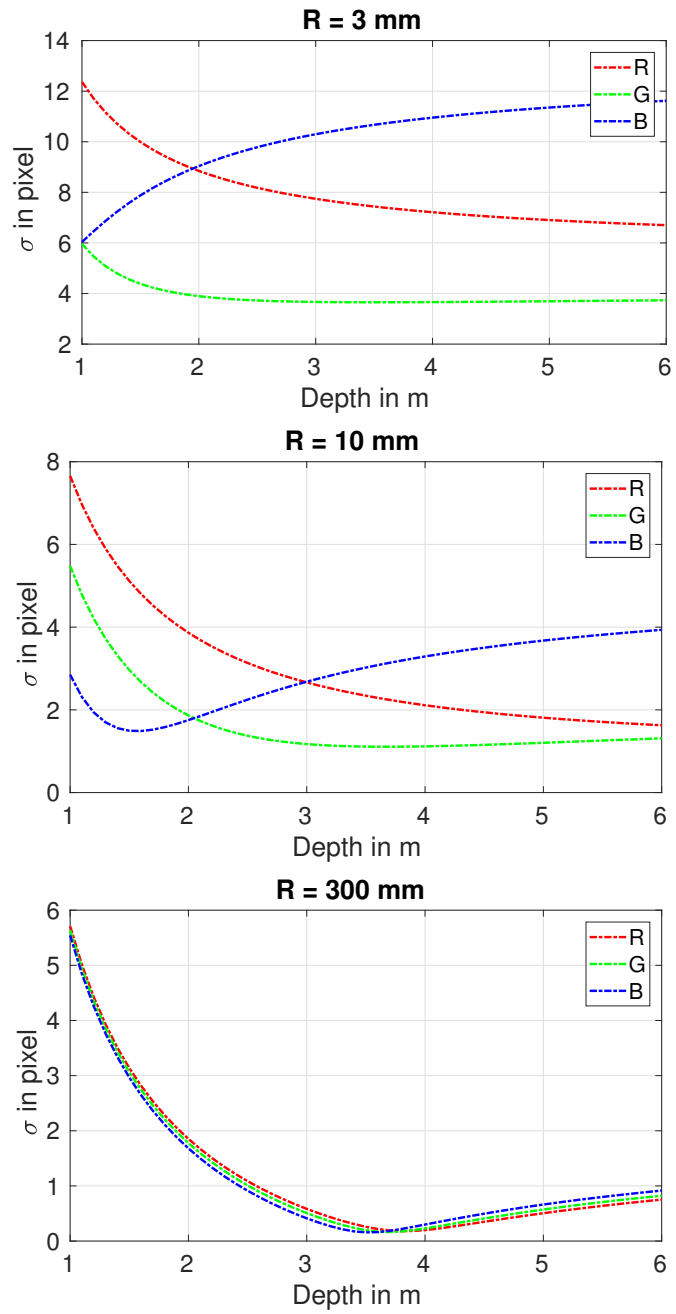

Fig. 9. Examples of variation of RGB PSF standard deviation taking into account intra-channel chromatic aberration, for different values of the radius of curvature of the chromatic add-on respectively $\mathrm{R}=3,10$ and $300 \mathrm{~mm}$.

\section{D.2. Depth estimation algorithm}

SIDFD from RGB images with chromatic aberrations is conducted with the method described in [7] and summarized in Appendix B). For each triplet of RGB patches extracted from the raw data, a PSF triplet is selected using a criterion derived within a maximum likelihood framework. Details concerning this algorithm are given in Appendix B. B. Potential PSF triplets were previously calibrated for each depth as described in the next section.

\section{D.3. PSF calibration}

With and without the add-on, the PSFs of each color channel are calibrated from 1 to $2.5 \mathrm{~m}$ with a step of $5 \mathrm{~cm}$, and from $2.5 \mathrm{~m}$ to

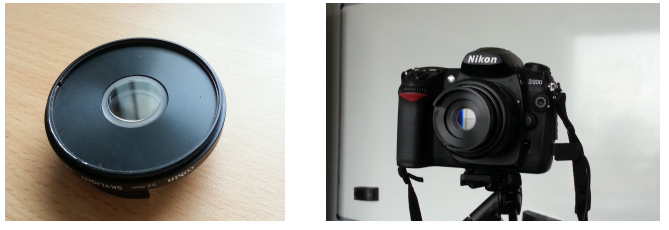

Fig. 10. Left: chromatic add-on put in the center of a mechanical mount. Right: camera with the chromatic add-on

$6 \mathrm{~m}$ with a step of $10 \mathrm{~cm}$, using acquisitions of a high frequency pattern [16]. Figure 11 shows examples of calibrated PSF at $4 \mathrm{~m}$ with the add-on. Chromatic aberration is visible with various defocus blur sizes in the RGB color channels.

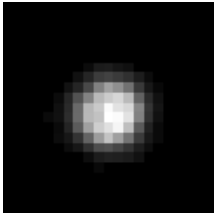

$\mathrm{R}$

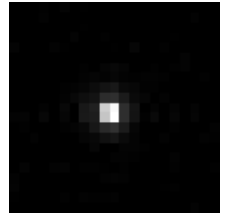

G

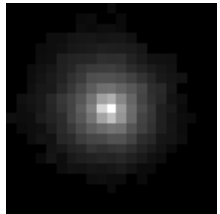

B
Fig. 11. RGB PSFs at $4 \mathrm{~m}$ with the chromatic add-on.

We experimentally measure the standard deviation $\sigma_{x}$ using a simple knife-edge blur estimation, proposed in [17], on black and white targets put at different distance from the camera. As with the cylinder add-on, direct comparison of these experimental values with theoretical $\sigma$ is not straightforward. However we show in Figure 12 that one can fit a set of parameters $f_{\#}, e$ and $f^{\prime}$ (see Appendix A), close to the expected values, that gives a good match between theoretical standard deviation and experimental measures of the blur. Those parameters are $f^{\prime}=35 \mathrm{~mm}$, $f_{\#}=3.9$ and $|e|=22 \mathrm{~mm}$.

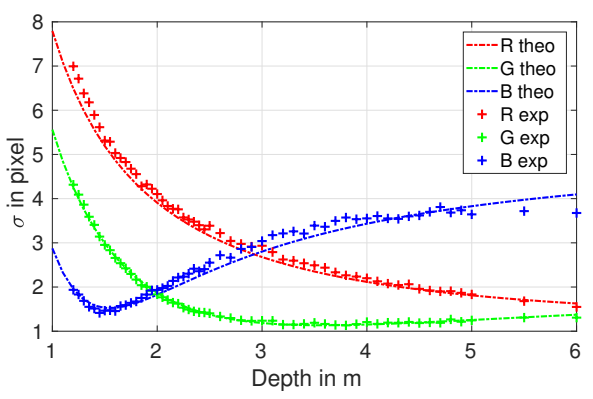

Fig. 12. Theoretical and experimental variations of $\sigma$ using the chromatic add-on. Theoretical curves are obtained with parameters : $f=35 \mathrm{~mm}, f_{\#}=3.9,|e|=22 \mathrm{~mm}$ and $R=10 \mathrm{~mm}$ and a focus at $3.6 \mathrm{~m}$ at $530 \mathrm{~nm}$.

\section{D.4. Experimental depth estimation}

Different textured patterns are put at a known distance from the camera. In both cases and at each depth, a depth map is estimated within a patch of $25 \times 25$ pixels. Figure 13 shows the mean depth estimation and standard deviation with respect to the ground truth, inside a centred region of the depth map corresponding to a square of $15 \times 15$ patches. We remove $5 \%$ of the maximal and minimal values in order to remove outliers in 
the statistics. With and without the add-on, depth estimation is unbiased in the range $1 \mathrm{~m}$ to $5 \mathrm{~m}$. However without the addon the MSE varies between 11 and $85 \mathrm{~cm}$ while it varies only between 8 and $40 \mathrm{~cm}$ with the add-on.
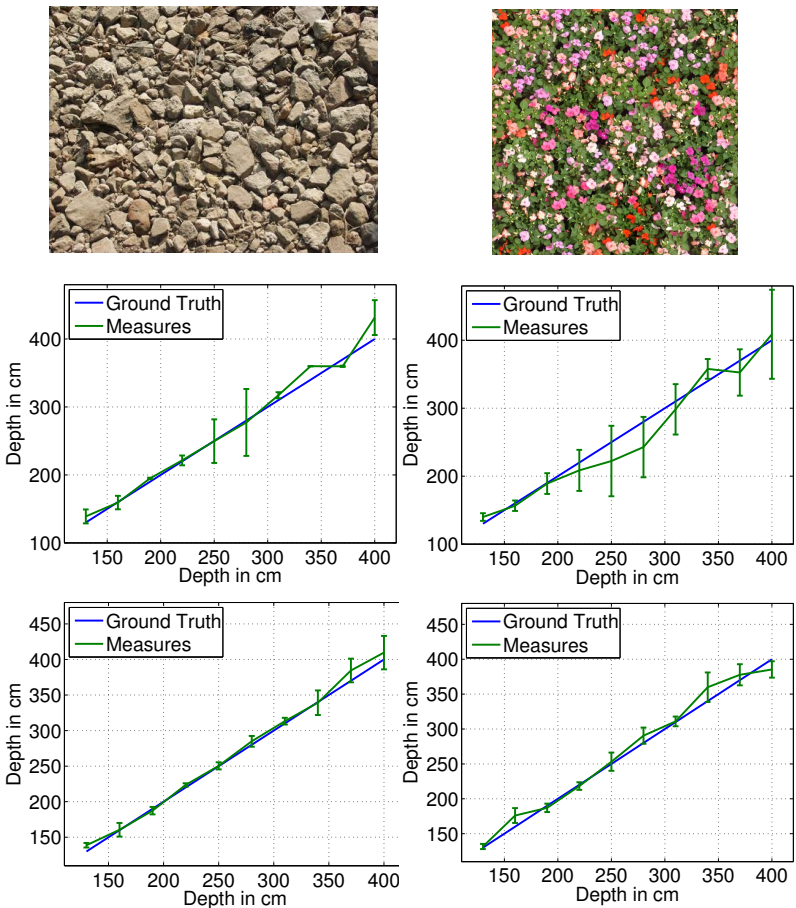

Fig. 13. Depth estimation experimental statistical results, without (middle row) and with (last row) the chromatic add-on.

\section{D.5. Depth maps}

Figure 14 shows two depth maps obtained with natural indoor and outdoor scenes.
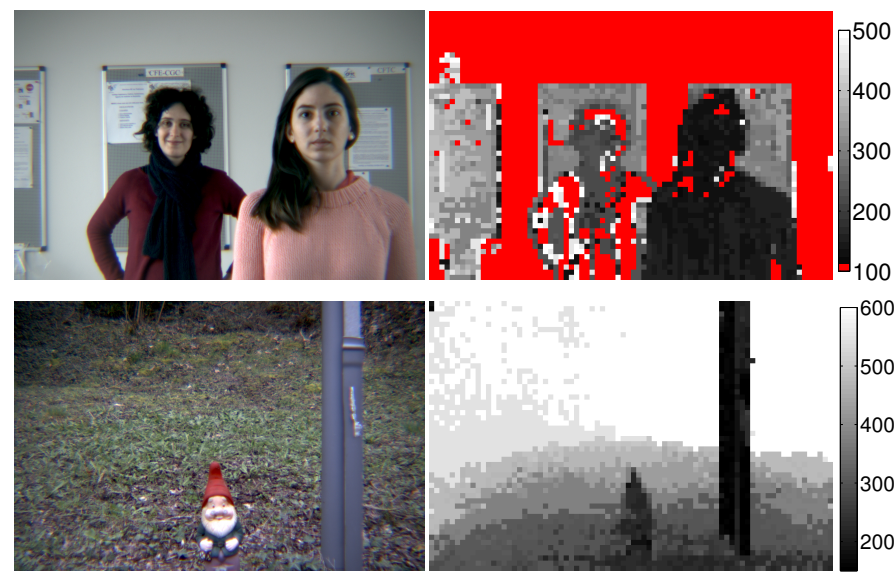

Fig. 14. Scenes and depth maps in $\mathrm{cm}$ for indoor and outdoor scenes. Red is for textureless regions corresponding to $\widehat{\alpha}>0.1$.

For both scenes the 3D profile within different parts of the scene are correctly restored. In the indoor scene, the estimation fails in textureless and low contrast regions, which is expected from any passive depth estimation technique. In contrast with the cylinder add-on, there is no aberrated values due to monodirectional regions.
Figure 15 shows depth maps obtained with natural indoor and outdoor scenes, with and without the add-on. As already mentioned, whatever the lens, passive depth estimation fails on untextured regions (shown in red). In textured regions, however, the improvement brought by the add-on is very significant. On the two leftmost scenes, the depth maps obtained without addon exhibit higher noise and several of outlier pixels wrongly located at the farthest or closest distance in the used range. The two scenes shown on the right also reveal that the results without add-on suffer from bias. The lower part of the stairs appear too close from the camera and do not exhibit the expected increase of the depth from one stair to the next. On the rightmost scene, the background wall is mislocated at $6 \mathrm{~m}$ from the camera, while it is actually much closer as shown in the results with an addon. These results confirm that the proposed chromatic add-on allows to reduce noise, outliers and bias on DFD.

\section{E. Applications}

Now that we have demonstrated the gain in 3D accuracy brought by a chromatic add-on for SIDFD, we present some applications. First, let us mention that while the depth maps presented in Figure 14 were quite noisy with outliers, they were stemming from a pixelwise decision on the depth value. Using spatial regularization methods one could get much better results, smoother within each object and with better delineation of foreground/background boundaries. As an illustration, we provide in Figure 16 the result of the matting method of [19] to the data of Figure 14. Using this type of regularized depth maps opens the way to several applications of the SIDFD using a camera equipped with an add-on. One could think of 3D scanning, man-device interaction, or 3D display.

Another application is refocusing, where a new image is generated from the original image and the depth map, with a different in-focus plane or an enlarged depth of field (the latter case is often reffered to as the "all-focus image"). In Figures 19 we present results of refocusing using a commercial camera and the chromatic add-on. To generate all-focus and refocused images, we first correct for lateral chromatic aberration. To do so we estimate an homography between the R,G and B channels and warp two channels onto the third. Then, denoising is applied using BM3D algorithm [20]. An all-focus image is generated using a high frequency transfer guided by the regularized depth maps of Figure 16. Figure 18 shows one example of such restoration processing. Details on this approach can be found in [7]. Note that the wrong depth values have sometimes propagated in textureless regions, however those regions will not create any artefacts in the refocused image, because textureless regions are insensitive to blur. Finally, we produce focused images at different in-focus plane positions using the all-focus image, the regularized depth map and the simple layer model presented in [21]. One can then simulate images from a camera with various optical settings in order to increase or decrease the depth of field. For instance, Figure 19 shows the results of refocused images obtained with the Nikon camera with parameters $f^{\prime}=20 \mathrm{~mm}$ and $f_{\#}=3$.

Chromatic add-ons can be used with various digital color camera, as long as raw RGB data can be extracted. For instance, Figure 20 shows an add-on designed to be used with a Nexus smartphone. Figures 21 and 22 show experimental image restoration and refocusing obtained with it. Raw data are extracted using a driver developed by the society Codra [22] based on the layer HAL3 of Android 5.0. 

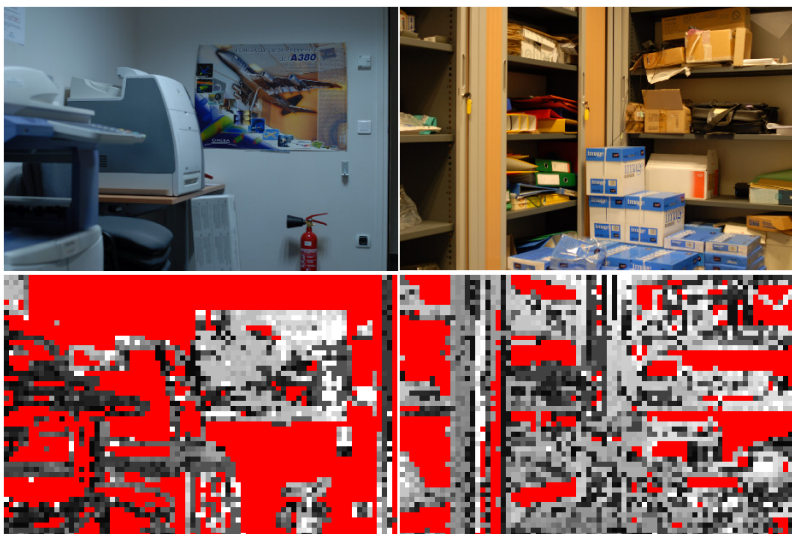

400
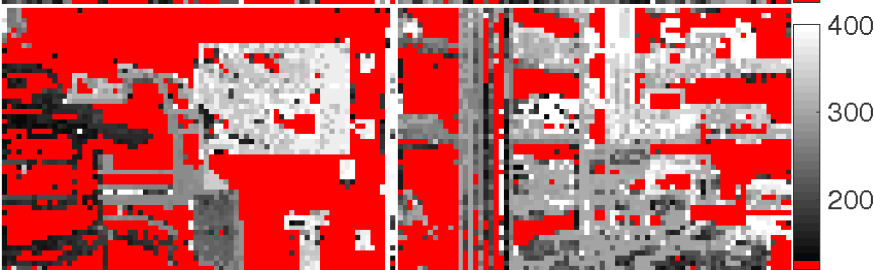

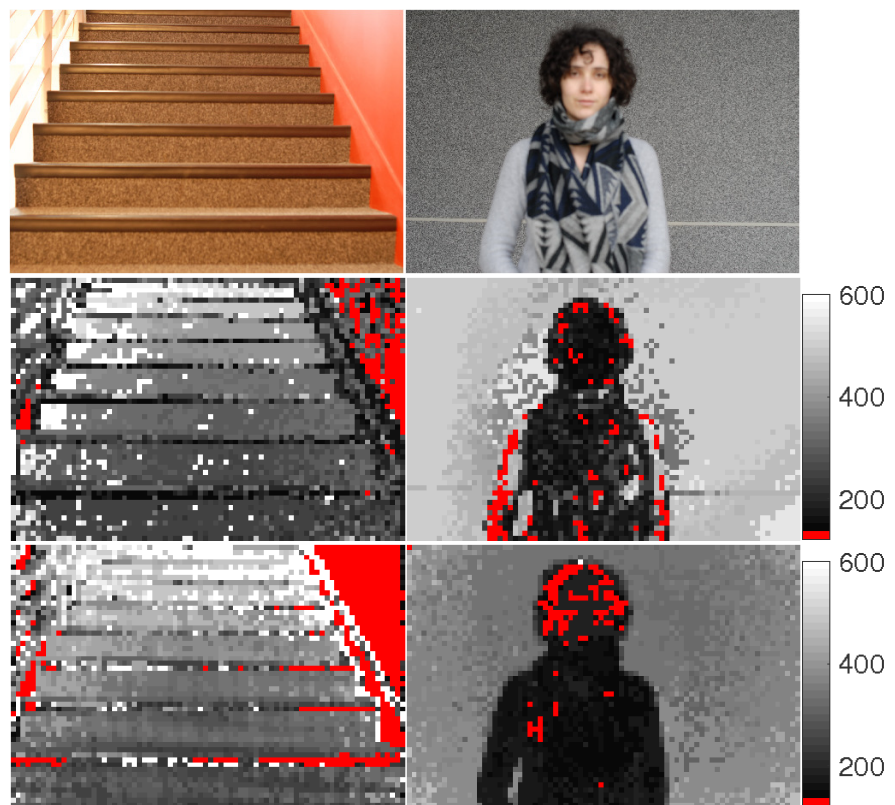

Fig. 15. Depth maps in $\mathrm{cm}$ for indoor and outdoor scenes. First row color images without the add-on. Second row : depth maps without the add-on, third row depth maps with the add-on. Red is for textureless regions corresponding to $\widehat{\alpha}>0.5$.
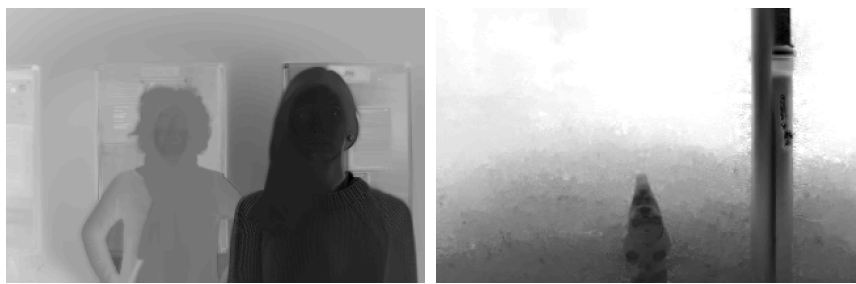

Fig. 16. Regularized depth maps using alpha matting [19] corresponding to the raw depth map of Figure 14.

\section{CONCLUSION}

In this paper we have presented two concepts of optical add-ons able to improve depth estimation ability of conventional cameras. The first one adds astigmatism using a cylinder lens and the second one adds chromatic aberration using a doublet of lenses having an identical refractive index only for a nominal wavelength. In contrast with previously proposed unconventional lenses for improved SIDFD, add-ons are cheap and easy to use. We have demonstrated the benefits brought by such add-ons in terms of depth estimation performance compared to conventional cameras. The cylinder add-on requires scenes with isotropic distributions of gradient orientations and leads to difficult image restoration issues. In our opinion, it would be suited to industrial applications where artificial texture can be added by painting or by controlled illumination. The chromatic add-on has less restrictions and can be used on outdoor or indoor situations, with any conventional lens. We have shown that it improves depth estimation and can be applied even for applications dealing with image quality such as image refocusing. Another interesting point about the presented add-ons is that their design depends essentially on a unique parameter, opening ways to efficient optimization process, depending on the application.

Further studies concern the optimization of the add-on with respect to depth estimation accuracy based on a theoretical per- formance model. It could be based on the performance model proposed in [23] which is a generalization of the model developed for a conventional lens in [8].

\section{A. PSF MODEL}

In this section we present the PSF model of an optical system made of a conventional lens with an add-on, combining diffraction, defocus and pixel integration. One could derive a fine modelisation from the knownledge of principal planes position, as well as input and output pupils positions (that can be measured experimentally) and using for instance Fourier Optics to simulate the PSF. We propose here a simpler model based on thin lens approximation and a Gaussian PSF model.

Both the add-on and the conventional lens are considered as perfect thin lens of focal length respectively $f_{a}^{\prime}$ and $f^{\prime}$, separated by the distance $|e|$. Figure 17 shows a scheme of both lenses and presents the main notations. We assume that the global system aperture is still the aperture of the conventional lens. Using the

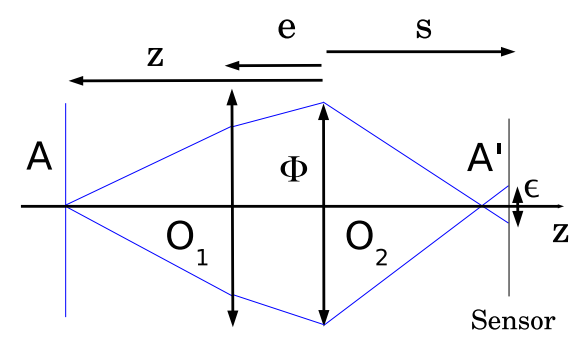

Add-on Conventional lens

Fig. 17. Schematic view of a hybrid optical system combining a conventional lens and an add-on.

thin lens law, the size of the defocus blur $\epsilon_{d e f}$ can be derived 
from the following equations :

$$
\begin{aligned}
\epsilon_{d e f} & =\Phi\left|\frac{s-\overline{O_{2} A^{\prime}}}{\overline{O_{2} A^{\prime}}}\right|, \\
\overline{O_{2} A^{\prime}} & =\frac{f^{\prime}\left(f_{a}^{\prime} z-e^{2}+z e\right)}{f^{\prime}\left(f_{a}^{\prime}-e+z\right)+\left(f_{a}^{\prime} z-e^{2}+z e\right)} .
\end{aligned}
$$

The PSF is modeled as a combined effects of the diffraction, geometric defocus and pixel integration. Assuming that each effect can be modeled as Gaussian PSF function this leads to a combined standard-deviation defined by :

$$
\sigma=\rho \sqrt{\left(2.44 \lambda f_{\#}(M+1)\right)^{2}+\epsilon_{d e f}^{2}+t_{p x}^{2} / 12}
$$

with $M$ the magnification, $\lambda$ the wavelength, $t_{p x}$ the pixel size. Parameter $\rho$ is a factor empirically fixed at 0.3. To simplify, we fix $M=s / z$ which means that we neglect the (slight) change of magnification due to the add-on.

\section{B. DEPTH FROM DEFOCUS ALGORITHM}

DFD consists in estimating depth from one or several defocused images. We note $n$ the number of images used to estimate the depth. In the general case, defocused images are modelled using a local convolution model. Let $\mathbf{Y}$ and $\mathbf{X}$ be respectively the concatenation of defocused image and scene patch vectors of size respectively $n N$ and $n M$. One can write :

$$
\mathbf{Y}=H_{z} \mathbf{X}+\mathbf{N}
$$

where $N$ stands for the noise process which is modeled as a zero mean white Gaussian noise (WGN). $H_{z}$ is the block diagonal matrix with each block corresponding to the convolution matrices of the PSFs relative to each image patch. Each PSF can be obtained by simulation or calibration. It is shown in $[7,15]$ that using a Gaussian prior on the scene parameterized by a precision matrix $\mathrm{D}$, the data marginal likelihood can be derived for a depth and SNR hypothesis. This leads to the following depth estimation criterion:

$$
\begin{aligned}
\widehat{k}, \widehat{\alpha} & =\arg \min _{k, \alpha} G L\left(z_{k}, \alpha\right) . \\
G L(z, \alpha) & =|P|_{+}^{-1 /(n N-m)} \mathbf{Y}^{t} P \mathbf{Y} .
\end{aligned}
$$

where $P=I-H_{z}\left(H_{z}^{t} H_{z}+\alpha D^{t} D\right)^{-1} H_{z}^{t}$ and $|A|_{+}$refers to the product of the non zero eigenvalues of a symmetric matrix $A$, with the eigenvalues raised to the power of their multiplicity, $m$ the number of the zero eigenvalues of $D$. Parameter $\alpha>0$ fixes the inverse SNR for the considered patch. $k$ is the index of depth within the finite set of potential depth values $\left\{z_{1}, \ldots, z_{k}, . . z_{K}\right\}$. Details on the implementation of the algorithm are given in [7, 15]. In the following we briefly describe the contents of $H_{z}$ and $D$ for the two cases considered in the paper.

\section{A. Application to single image DFD}

In this case, $n=1$ and $\mathbf{Y}$ (respectively $\mathbf{X}$ ) simply collects the $N$ (resp. the $M$ ) pixels of the image (resp. scene) patch in the lexicographical order. Matrix $H_{z}$ is directly a convolution matrix which depends on the PSF at depth $z$. As in [8, 15], matrix $D$ is the concatenation of the convolution matrix relative to horizontal and vertical first order derivative kernels [-1 1] and $[-11]^{t}$, which amounts to considering a Gaussian prior on the scene gradients. This matrix as a single zero eigenvalue $(m=1)$ corresponding to homogeneous patch.

\section{B. Application to DFD with chromatic aberration}

In this case, $n=3, \mathbf{Y}=\left[y_{R}^{t} y_{G}^{t} y_{B}^{t}\right]^{t}$, and $\mathbf{X}=\left[x_{R}^{t} x_{G}^{t} x_{B}^{t}\right]^{t}$, where for each channel $c, y_{c}$ (respectively $x_{c}$ ) collects $N$ (resp. $M$ ) pixels of the image (resp. scene) patch in the lexicographical order. Given that color channels are highly correlated, vector $\mathbf{X}$ is decomposed into luminance and chrominance components. A separable Gaussian prior can then be used for the scene assuming that luminance and chrominance are decorrelated. $D$ is then a block diagonal matrix whose diagonal elements are the concatenation of the convolution matrix relative to horizontal and vertical first order derivative kernels $[-11]$ and $[-11]^{t}$, which amounts to considering a Gaussian prior on the luminance and chrominance gradients. Note that the prior matrix $D$ incorporates a parameter that models the ratio between the gradient variances of the luminance and the chrominance components. Matrix $H_{z}=H_{z}^{C} T \otimes I_{M}$ where $I_{M}$ is the identity matrix of size $M \times M$ and $T$ the matrix transforming luminance and chrominances data to RGB data. $H_{z}^{C}$ is the block diagonal matrix with each block corresponding to the convolution matrix of the PSF at depth $z$ relative to each colour channel. Finally, the number of eigenvalues of $D$ is $m=3$, for homogeneous patches in the three luminance and chrominances components. Details on this algorithm can be found in [7].

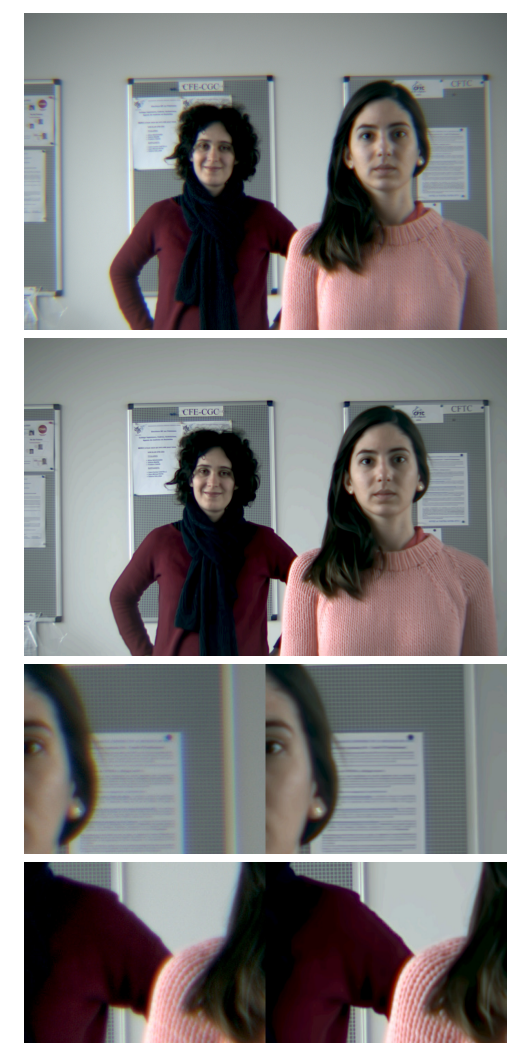

Fig. 18. Top: Raw image from the Nikon D200 and the chromatic add-on. Middle: Restored all-focus image. Bottom: Zoomed image portion : raw (left) and restored image (right).

\section{REFERENCES}

1. https://www.stereolabs.com/.

2. https://www.ptgrey.com/bumblebee-xb3-1394b-stereo-vision-camerasystems-2.

3. https://software.intel.com/en-us/realsense/r200camera. 

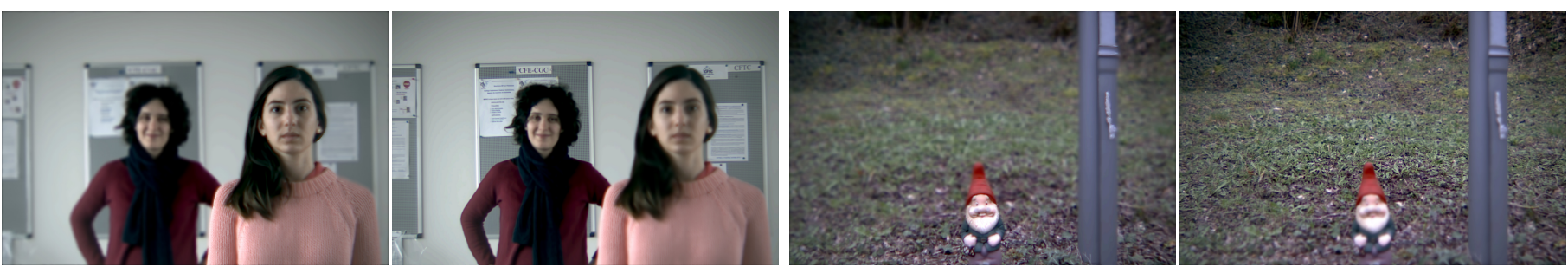

Fig. 19. Examples of refocused images using the chromatic add-on and the Nikon D200 with a $35 \mathrm{~mm}$ lens.
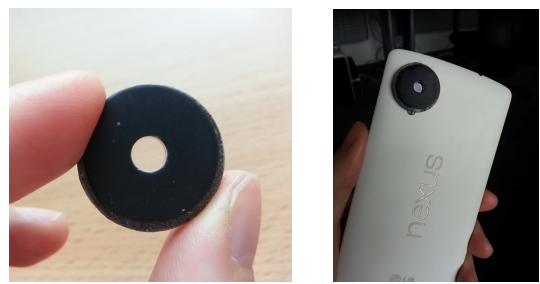

Fig. 20. Chromatic add-on designed for a Nexus 5.
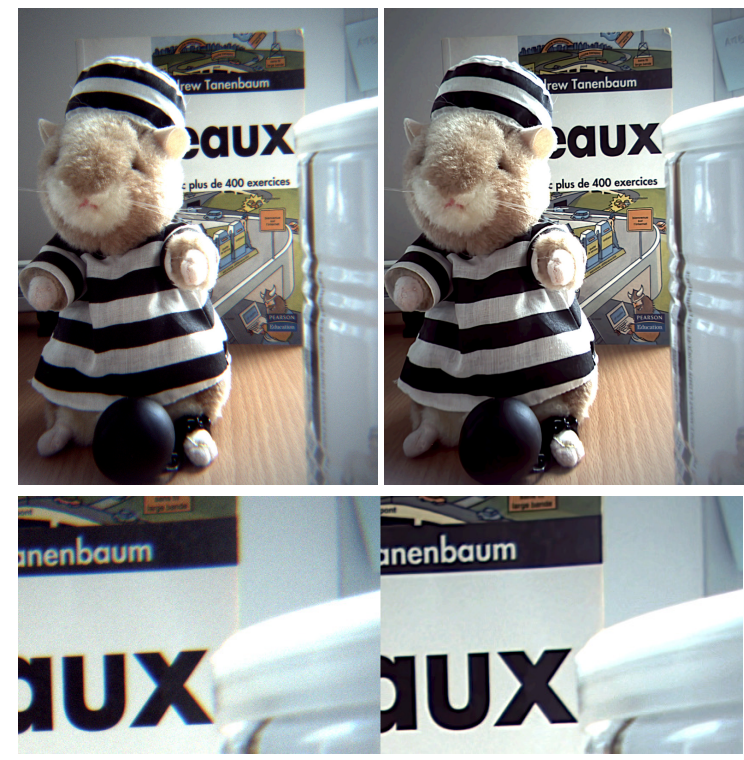

Fig. 21. Top row : Original image taken with the Nexus 5 and the chromatic add-on (left), all-focus image after image restoration (rigth). Bottom row : Zoomed on some image portions (left : raw image, right: corrected image).
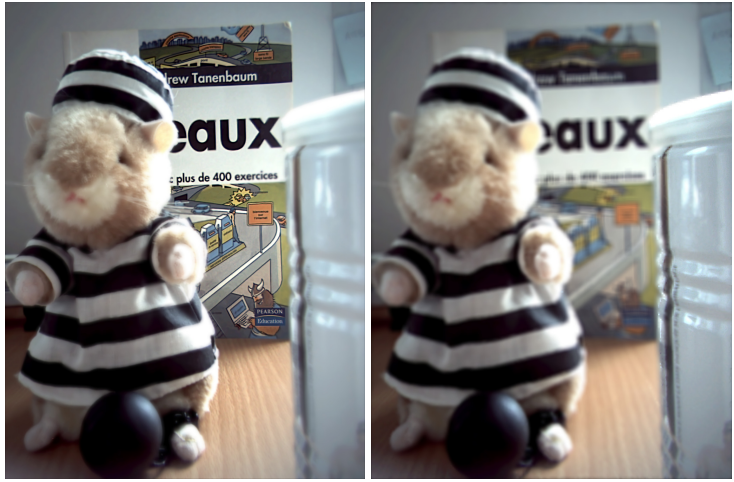

Fig. 22. Examples of refocused images with the smartphone Nexus 5 and a chromatic add-on.
4. A. Pentland, "A new sense for depth of field," IEEE Transactions on Pattern Analysis and Machine Intelligence 4 (1987).

5. M. Subbarao, "Parallel depth recovery by changing camera parameters," in "Internation Conference on Computer Vision," (1988).

6. S. Pasinetti, I. Bodini, M. Lancini, F. Docchio, and G. Sansoni, "A depth from defocus measurement system using a liquid lens objective for extended depth range," IEEE Transactions on Instrumentation and Measurement 66, 441-450 (2017).

7. P. Trouvé, F. Champagnat, G. Le Besnerais, J. Sabater, T. Avignon, and J. Idier, "Passive depth estimation using chromatic aberration and a depth from defocus approach," Applied Optics 52, 7152-7164 (2013).

8. P. Trouvé-Peloux, F. Champagnat, G. Le Besnerais, and J. Idier, "Theoretical performance model for single image depth from defocus," Journal of the Optical Society of America A 31, 2650-2662 (2014).

9. A. Levin, R. Fergus, F. Durand, and W. Freeman, "Image and depth from a conventional camera with a coded aperture," ACM Transactions on Graphics (TOG) 26, 1-9 (2007).

10. A. Chakrabarti and T. Zickler, "Depth and deblurring from a spectrally varying depth of field," in "European Conference on Computer Vision," (2012).

11. A. Sellent and P. Favaro, "Optimized aperture shapes for depth estimation," Pattern Recognition Letters 40, 96-103 (2014).

12. E. Dowski Jr and W. Cathey, "Single-lens single-image incoherent passive-ranging systems," Applied Optics 33, 6762-6773 (1994).

13. A. Greengard, Y. Y. Schechner, and R. Piestun, "Depth from diffracted rotation," Optics letters 31, 181-183 (2006).

14. C. Cierpka, M. Rossi, R. Segura, and C. Kähler, "On the calibration of astigmatism particle tracking velocimetry for microflows," Measurement Science and Technology 22, 15401-15410 (2010).

15. P. Trouvé, F. Champagnat, G. L. Besnerais, and J. Idier, "Single image local blur identification," in "IEEE International Conference on Image Processing," (2011).

16. M. Delbracio, P. Musé, A. Almansa, and J.-M. Morel, "The nonparametric sub-pixel local point spread function estimation is a well posed problem," International Journal of Computer Vision 96, 175-194 (2012).

17. S. E. Reichenbach, S. K. Park, and R. Narayanswamy, "Characterizing digital image acquisition devices," Optical Engineering 30, 170-178 (1991).

18. J. Jiang, D. Liu, J. Gu, and S. Susstrünk, "What is the space of spectral sensitivity functions for digital color cameras?" in "2013 IEEE Workshop on Applications of Computer Vision (WACV)," (2013), pp. 168-179.

19. S. Zhuo and T. Sim, "On the Recovery of Depth from a Single Defocused Image," Computer Analysis of Images and Patterns pp. 889-897 (2009).

20. K. Dabov, A. Foi, V. Katkovnik, and K. Egiazarian, "Image denoising by sparse 3-d transform-domain collaborative filtering," IEEE Transactions on Image Processing 16, 2080-2095 (2007).

21. S. W. Hasinoff and K. N. Kutulakos, "A layer-based restoration framework for variable-aperture photography," in "IEEE International Conference on Computer Vision," (2007).

22. https://fr.codra.net/informatique/.

23. P. Trouvé, F. Champagnat, G. Le Besnerais, G. Druart, and J. Idier, "Design of a chromatic 3d camera with an end-to-end performance model approach," in "IEEE Conference on Computer Vision and Pattern Recognition (CVPR) Workshops," (2013). 